\title{
WORKFORCE Factors affecting individual task prioritisation in a workplace setting
}

\author{
Authors: Sophie Middleton, ${ }^{A}$ Alexandra Charnock, ${ }^{B}$ Sarah Forster ${ }^{C}$ and John Blakey
}

Task prioritisation (TP), the ability to arrange assigned responsibilities in order of importance, has been identified by doctors as the most important non-technical skill when working out of hours. This review aimed to identify the factors influencing task prioritisation in a workplace setting. The following databases were searched for relevant studies: BNI, CINAHL, EMBASE, MEDLINE and PsycINFO as well as the reference lists of relevant articles, review papers and Google Scholar. Data were extracted from the studies using a standard proforma. Twenty relevant papers were identified from management, aviation and healthcare fields. Task prioritisation ability differed across individuals and was associated with personality and experience. Tasks were prioritised based on length, urgency, importance and reward. With practice people become more efficient at task prioritisation. Practical applications include education, personalised task management software and intrusive notifications. Additional research is needed to assess the effects of such interventions in a healthcare setting.

KEYWORDS: Task prioritisation, out-of-hours, non-technical skills, systematic review, medical education

\section{Introduction}

Hospitals in the UK are receiving a rising number of admissions year on year. ${ }^{1}$ These admissions are of increasingly multimorbid individuals for whom an ever-greater array of tests and treatments are available. At the same time, total bed numbers are falling resulting in pressure to reduce length of stay. ${ }^{2}$ An increasing amount of clinical work is therefore delivered in the out-ofhours $(\mathrm{OOH})$ period: outside of 9 am to $5 \mathrm{pm}$, Monday to Friday. During $\mathrm{OOH}$ periods, care is delivered by a smaller workforce with proportionally more junior staff. ${ }^{3}$ The volume and nature of clinical work now expected of junior doctors tests their non-technical skills, such as communication and time management, and is a source of significant stress. ${ }^{4,5}$ These non-technical skills can impact on

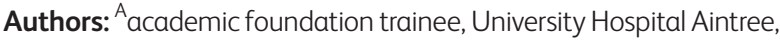
Liverpool, UK; ${ }^{B}$ foundation year 2 trainee, University Hospital Aintree, Liverpool, UK; ' NIHR academic clinical fellow, University of Nottingham School of Medicine, Nottingham, UK; ${ }^{\text {D }}$ respiratory consultant, Royal Liverpool and Broadgreen University Hospitals NHS Trust, Liverpool, UK quality of care ${ }^{6}$ and are a potential source of adverse events, such as during the annual junior doctor changeover. ${ }^{7}$

Task prioritisation (TP) is the ability to arrange or deal with assigned responsibilities in order of importance and is considered a non-technical skill. It involves a variety of abilities such as: the creation of an initial agenda; regular assessment of a situation; initiation and monitoring of tasks; allocating resources in order of priority and interrupting low-priority tasks when urgent tasks arise. ${ }^{8}$ Task prioritisation has been identified by doctors and medical students as the most important non-technical skill when working $\mathrm{OOH}^{4}$ However, its importance is not reflected in the amount of research conducted and there is little emphasis on this skill through undergraduate medical training. ${ }^{4}$

Improving formal training in TP for undergraduates and junior doctors will require a sound theoretical framework and an evidence base. These will be informed by existing research in other fields, as has been the case for other aspects of quality and safety improvement such as aviation and surgical checklists. ${ }^{9}$ For example, the Cockpit Task Management approach was devised by Funk and colleagues in $1991 .{ }^{8}$ It is the process by which pilots choose which task to attend to and has been used to minimise human error in critical situations. ${ }^{8}$ Other theories allocate human biases, attention, perception and memory as key influencers of behaviours. ${ }^{10,11}$ Findings from studies in other fields also have the potential to positively effect change in the working environment, eg enabling the presentation of information in a manner that facilitates sound decision making around prioritisation.

\section{Aims}

This review aims to identify studies describing the methods people employ to prioritise tasks and the factors that influence their choices that are applicable to a workplace setting. It relates these findings to opportunities to support improvements in $\mathrm{OOH}$ clinical practice.

\section{Method}

\section{Search strategy}

The following databases were searched on 3 January 2018 for relevant studies: BNI (British Nursing Index), CINAHL (Cumulative Index to Nursing and Allied Health Literature), EMBASE (Excerpta Medica Database), Medline and PsycINFO. The literature search used the following terms (including English and American-English spellings): 'task prioritisation' and 'task management'. The searches were not limited by study type, date of publication or language. A Google 


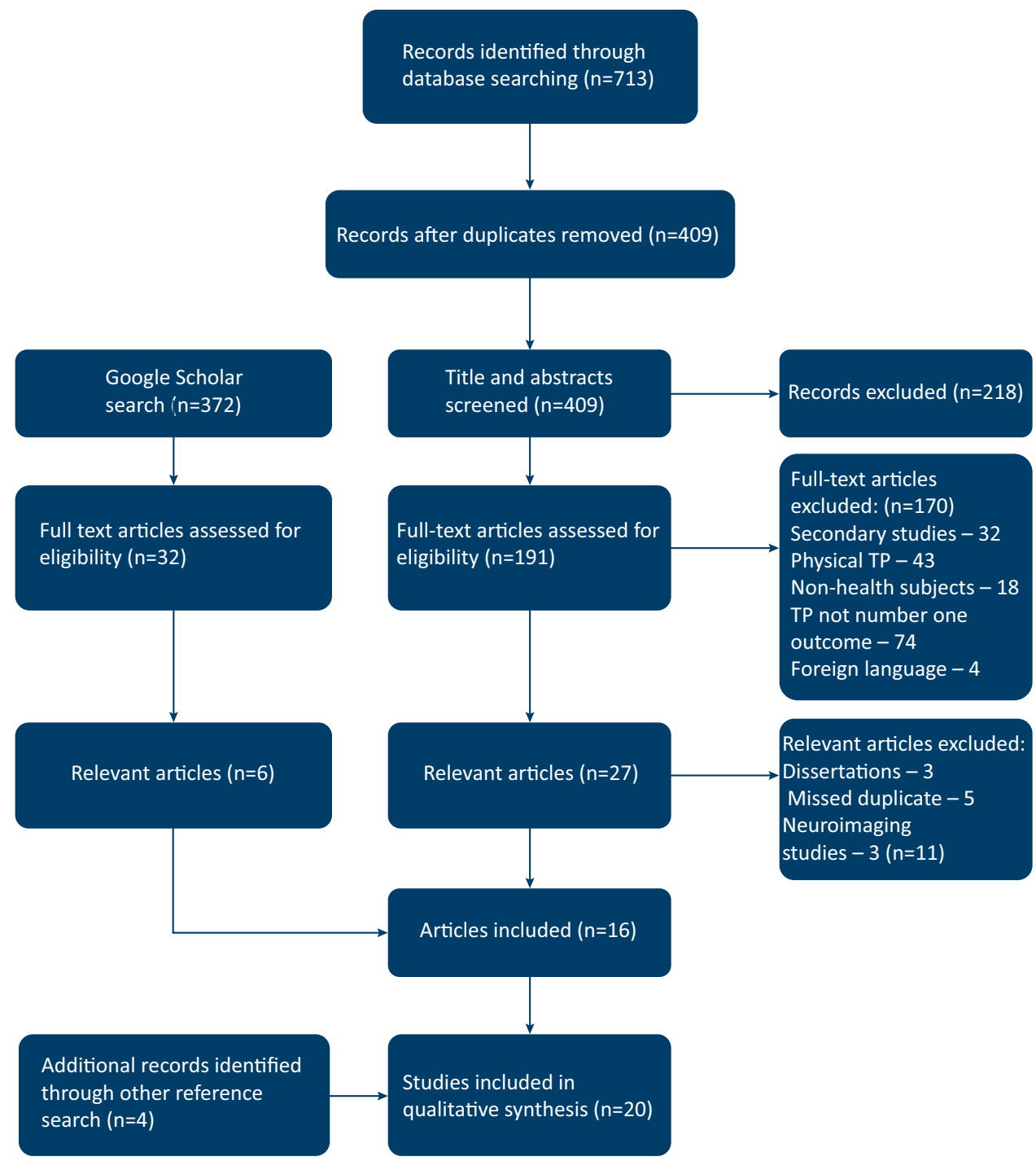

Fig 1. Paper selection process.

Scholar search was also undertaken on 11 December 2017 which used the following terms: 'workplace 'task prioritisation' OR ‘task prioritization' -children -walking'. The reference list of relevant articles as well as review papers were also searched to identify further studies.

\section{Selection criteria}

Papers were selected in a three-step process: removal of duplicates, abstract screening to remove obviously non-contributory papers and full paper review (Fig 1). Initial data extraction was undertaken by a single researcher. Of the selected papers from the database search, $50 \%$ underwent data extraction in duplicate by one of two independent researchers as quality assurance. We included all primary studies that had a task management intervention or had a measure of task prioritisation as a primary outcome.

\section{Data extraction}

Data were extracted from the studies using a proforma which included: author, title and date of publication; study population; study objectives; study intervention and study outcome (see Supplementary file S1).

\section{Assessment of quality}

Methodological quality was assessed using the Joanna Briggs Institute Critical Appraisal Checklist for Analytical Cross Sectional Studies. ${ }^{12}$ Where papers contained more than one experiment, quality was assessed based on all studies meeting the criteria. Unpublished dissertations were not included.

\section{Results}

\section{Search results}

The database search identified 713 results comprising 409 unique papers after duplicates were removed. After abstracts were read to exclude irrelevant papers and a Google Scholar search conducted, 223 full papers were reviewed. 
Twenty relevant publications covering 30 studies from the USA, China and Europe were identified from the database, Google Scholar and reference search, of which 9 related to management, 6 aviation, 4 healthcare and 1 driving. Of these studies, 13 involved direct observation of tasks, 5 involved interviews and 5 involved simulation. Of the observational studies, $6(46.2 \%)$ were in a workplace setting and the remainder were purely experimental.

\section{Individual level features associated with task prioritisation}

Nine papers considered individual factors which influence TP behaviours. Workplace-based observational studies of nurses found that previous experiences such as medication mistakes influence how you prioritise a task. ${ }^{13}$ Those more experienced were better at prioritising tasks ${ }^{14}$ and less likely to let lower priority tasks interrupt them. ${ }^{14,15}$ Gan et al found that people who are more proactive in preparing for future events were less likely to favour immediate rewards. ${ }^{16}$ They were also more likely to demonstrate preferences for urgent but less important tasks. ${ }^{16}$

Office-based studies of personal task management concluded users have a preset tendency on how they organsise tasks. ${ }^{17,18}$ This includes either using a general TP tool such as an online calendar, or adopting an existing tool to suit personal preferences. ${ }^{17}$ During driving and flight simulation people also followed individual TP preferences ${ }^{19,20}$ despite instruction otherwise. ${ }^{19}$ Similarly, Arrington et al found study participants tended to favour task orders they had previously adopted when similar options were presented again. ${ }^{21}$ No studies looked at the effect of age, gender or intelligence on TP strategies in the workplace.

\section{Task factors influencing TP}

Nine papers looked at the task-related factors which influence TP. Two flight simulator studies looking at cockpit task management found that; resource requirements, time, effort, complexity, procedure, importance, urgency and salience created significant effects on task prioritisation. ${ }^{20,22}$ Procedure was the most influential factor among airline pilots ${ }^{20}$ and tasks that were shorter were less likely to be interrupted. ${ }^{23}$ The driver simulator study found preference for the more rewarding task. ${ }^{19}$

In observational studies, participants were more likely to choose easier and shorter tasks. ${ }^{23-25}$ They were also more likely to prioritise those tasks subjectively deemed salient ${ }^{26}$ and those that required a large amount of working memory were less likely to be interrupted. ${ }^{25}$ Whereas in a healthcare setting, nurses prioritised clinical emergencies, high uncertainty activities and core clinical caregiving over personal breaks, documentation and social interaction. $^{13,27}$

\section{Non-task factors that influence prioritisation}

Nine papers mentioned non-task related factors influencing prioritisation. The intrusiveness of a prompt such as audio-visual cues and tunnel displays increased the likelihood that a current task would be interrupted among airline pilots. ${ }^{28}$ However, pilots were much slower to acknowledge an event when the ongoing activity was auditory as compared to visual. ${ }^{23}$ The origin of the task also appears signifcant: internet users chose to interrupt a task based on notification source, ${ }^{29}$ nurses prioritise tasks from patients and seniors ${ }^{13,15}$ and airline pilots prioritise communications from air traffic control and dispatch over flight attendants and cabin chimes. ${ }^{30}$

The working enviroment also impacts on task management. The dermination of the priority of a task among nurses was found to be a dynamic process based on its context within fluid situations. ${ }^{13,15}$ In high stress scenarios people were more likely to choose shorter tasks with more immediate rewards to alleviate the stressful mood and create a positive mood and sense of accomplishment. ${ }^{16}$ When combined with task complexity, the number of concurrent tasks had significant effects on ability to prioritise tasks. ${ }^{22}$ Although alerts encourage task switching, receiving alerts degrade primary task performance. ${ }^{24}$

\section{Task prioritisation training}

Two papers considered training to improve task prioritisation using a multitasking software called Tardast. ${ }^{31,32}$ This computer programme was based on the theory of maintaining several spinning plates with multiple simulated tasks being controlled by the participants at any time. Initially, participants handled too many tasks that were unmaintainable but with practice the majority of participants learned to handle fewer tasks (or ignore more tasks). This resulted in a better performance but did not match the optimum task performance of the computer model. ${ }^{32}$

Feedback also affected multitasking performance. In the second Tardast study participants either recieved feedback on each task (local) or on all the tasks as a whole (global). Participants recieving local feedback paid more attention to the feedback display than those receiving global feedback therefore allowing better control of the multitasking system than global outcome feedback. ${ }^{31}$ This was further replicated in an observational study which found that higher quality, immediate feedback on a task led participants to place higher salience, time and effort on that task. ${ }^{26}$

\section{Discussion}

Twenty relevant papers were identified from a wide range of fields including management, aviation and healthcare. Prioritisation was based on task-related factors such as length, urgency, importance, procedure and reward and non-task-related factors such as intrusiveness, context, source and stress. Individuals have a preset bias in how they organise tasks but with practice people can become more efficient at task prioritisation. While there were many studies looking at task management as a non-technical skill there were very few studies looking at how people prioritise tasks and even fewer in a workplace setting. The studies in this systematic review were of variable quality (Supplementary file S2) and in a relatively small sample size; therefore, results may not be representative.

Perhaps the most striking conclusion from this review is the lack of relevant literature for such an important topic. Task prioritisation is a vital skill for all healthcare clinicians and while these studies give us an insight into theorectical task prioritisation, healthcare is a dynamic multitasking environment in which tasks change in urgency over time. Hence it is unclear how these theories can apply to an unstable prioritising setting. Further complicating TP in healthcare is the added layer of location: staff working $\mathrm{OOH}$ may cover a large clinical area, and so travel time and routing are important factors to weight when making TP decisions. 


\section{Limitations}

This was a systematic review based on a search of a wide number of databases and papers. The selection criteria focused on TP in a practical setting and therefore theoretical papers may have been missed. Papers were selected by a proforma and two independent reviewers to avoid selection bias. However, only $50 \%$ of papers were reviewed by a second person; ideally this would have been $100 \%$. All levels of evidence were considered including abstracts and dissertations to avoid inclusion bias. Only papers written in English were included potentially excluding relevant studies.

\section{Next steps}

Working conditions in the NHS have become increasingly pressurised, ${ }^{1,3,4}$ leading to increasing stress among doctors. ${ }^{5}$ This, coupled with increasing workload, could potientially be creating an environment in which TP is impaired. ${ }^{16,22}$ Although stress is almost impossible to eliminate in the healthcare system, consideration needs to be given to how this can effect the non-technical skills of doctors. In particular, ensuring safe staffing levels and adequate breaks.

Task prioritisation is personalised process; ${ }^{16-19}$ therefore, any task management software must have the ability to be individualised. Previous $\mathrm{OOH}$ s software include Nerve Centre, a program that allows task request, allocation and management on handheld devices for $\mathrm{OOH}$ care. ${ }^{33}$ This was well received by users and shows potential for wireless techology to be introduced in hospitals. Combining this technology with personalised task management software that allows individualisation would enable users to optomise their task management procedures.

The way tasks are presented also needs to be considered in any paperless system. Multiple studies have documented that many clinicians experience 'alarm fatigue' with multiple alerts and false alarms leading them to ignore alarms over time. ${ }^{34}$ Our review has mentioned that notification source ${ }^{13,15,29,30}$ and the intrusiveness of the notification eg audio-visual alerts ${ }^{28}$ effect the prioritisation of the task. Combining this with $\mathrm{OOHs}$ software could enable a system that can encourage the user to prioritise tasks as necessary by altering type of notification to task priority.

Task prioritisation can be improved upon with practice. This not only confirms previously held knowledge ${ }^{4}$ but also supports the argument for training at undergraduate and postgraduate levels. Current training has moved away from apprentiship practices to check-box competencies leaving medical students feeling underprepared for working life. ${ }^{5}$ Further development of shadowing provision for medical students or simulation for postgraduates could aid non-technical skills in junior doctors. An example of this already in place is NightShift, an online simulation of a night shift trialled to improve the non-technical skills of newly qualified doctors. ${ }^{35}$ Preliminary studies have shown a need for more engaging ways to teach non-technical skills. ${ }^{35}$

\section{Conclusion}

Most studies relating to TP were theoretical and outside the healthcare setting hence real world application is unclear. The principles discovered include individual, task and non-task related factors. Practical applications include undergraduate and postgraduate education, personalised task management software and intrusive notifications. Additional research is needed to assess the effects of such interventions in a healthcare setting.

\section{Supplementary material}

Additional supplementary material may be found in the online version of this article at http://futurehospital.rcpjournal.org:

S1 - Extracted data

S2 - Methodological quality assessment

\section{References}

1 Secondary Care Analysis Team, NHS Digital. Hospital admitted patient care activity 2015-16. Health and Social Care Information Centre. NHS Digital, 2016.

2 Ewbank L, Thompson J, McKenna H. NHS hospital bed numbers: past, present, future. The King's Fund, 2017.

3 Brown M, Syrysko P, Sharples S et al. Developing a simulator to help junior doctors deal with night shifts. Contemporary Ergonomics and Human Factors 2013:289-96.

4 Brown M, Shaw D, Sharples S, Jeune IL, Blakey J. A survey-based cross-sectional study of doctors' expectations and experiences of non-technical skills for out of hours work. BMJ Open 2015;5: e006102.

5 British Medical Association. Cohort study of 2006 medical graduates - Tenth report. London: BMA, 2016.

6 Maggs F, Mallet M. Mortality in out-of-hours emergency medical admissions - more than just a weekend effect. J $R$ Coll Physicians Edinb 2010;40:115-8.

7 Jen MH, Bottle A, Majeed A, Bell D, Aylin P. Early in-hospital mortality following trainee doctors' first day at work. PloS One 2009;4:e7103.

8 Funk K. Cockpit task management: Preliminary definitions, normative theory, error taxonomy, and design recommendations. The International Journal of Aviation Psychology 1991;1:271-85.

9 Haynes AB, Weiser TG, Berry WR et al. A surgical safety checklist to reduce morbidity and mortality in a global population. $N$ Engl ] Med 2009;360:491-9.

10 Wickens CD. Multiple resources and mental workload. Human Factors 2008;50:449-55.

11 Wickens CD, McCarley JS. Applied attention theory. CRC press; 2007.

12 Moola S, Munn Z, Tufanaru C et al. Systematic reviews of etiology and risk. In: Aromataris E, Munn Z (eds). Joanna Briggs institute reviewer's manual. The Joanna Briggs Institute, 2017.

13 Colligan L, Bass EJ. Interruption handling strategies during paediatric medication administration. BMJ Qual Saf 2012;21:912-7.

14 Koh RY, Park T, Wickens CD. An investigation of differing levels of experience and indices of task management in relation to scrub nurses' performance in the operating theatre: analysis of videotaped caesarean section surgeries. Int ] Nurs Stud 2014;51:123040.

15 Koh RYI, Tay BTC, Yang X, Donchin Y, Helander M. Cognitive factors influencing the management of interruptions during surgical counts. Proceedings of the Human Factors and Ergonomics Society Annual Meeting 2011;55:680-4.

16 Gan Y, Wang Y, Meng R et al. Temporal discounting mechanisms of future oriented coping: Evidence from delay discounting and task prioritization paradigms. Journal of Behavioral Decision Making 2015;28:529-41.

17 Haraty M, McGrenere ], Tang C. How personal task management differs across individuals. Int J Hum Comput Stud 2016;88:13-37.

18 Bellotti V, Dalal B, Good N, Flynn P, Bobrow DG, Ducheneaut N. What a to-do: Studies of task management towards the design of a personal task list manager. Proceedings of the SIGCHI conference on Human factors in computing systems 2004:735-42.

19 Jansen RJ, van Egmond R, de Ridder H. Task prioritization in dual-tasking: Instructions versus preferences. PloS One 2016;11: e0158511. 
20 Colvin K, Funk K, Braune R. Task prioritization factors: Two part-task simulator studies. The International Journal of Aviation Psychology 2005; 15:321-38.

21 Arrington CM, Weaver SM, Pauker RL. Stimulus-based priming of task choice during voluntary task switching. J Exp Psychol Learn Mem Cogn 2010;36:1060-7.

22 Chou C, Madhavan D, Funk K. Studies of cockpit task management errors. The International Journal of Aviation Psychology 1996;6:307-20.

23 Damos DL, Tabachnick BG. Cockpit task prioritization: Jumpseat observations. Los Angeles: Damos Research Associates, 2001.

24 Duggan B, Johnson H, Sørli P. Interleaving tasks to improve performance: users maximise the marginal rate of return. Int J Hum Comput Stud 2013;71:533-50.

25 Gutzwiller RS, Wickens CD, Clegg BA. The role of time on task in multi-task management. J Appl Res Mem Cogn 2016;5:176-84

26 Northcraft GB, Schmidt AM, Ashford SJ. Feedback and the rationing of time and effort among competing tasks. J Appl Psychol 2011;96:1076.

27 Patterson ES, Ebright PR, Saleem J]. Investigating stacking: How do registered nurses prioritize their activities in real-time? Int J Ind Ergon 2011;41:389-93.

28 Iani $C$, Wickens $C D$. Factors affecting task management in aviation. Human factors 2007:49:16-24.

29 Paul CL, Komlodi A, Lutters W. Interruptive notifications in support of task management. Int J Hum Comput Stud 2015;79:20-34.
30 Damos DL. The effect of interruptions on part 121 air carrier operations. NASA 1998.

31 Neth H, Khemlani SS, Gray WD. Feedback design for the control of a dynamic multitasking system: Dissociating outcome feedback from control feedback. Human factors 2008;50:643-51.

32 Shakeri S, Funk K. A comparison of human and near-optimal task management behavior. Human factors 2007:49:400-16.

33 Blakey JD, Guy D, Simpson C et al. Multimodal observational assessment of quality and productivity benefits from the implementation of wireless technology for out of hours working. BMJ Open 2012;2:e000701.

34 Ruskin KJ, Hueske-Kraus D. Alarm fatigue: Impacts on patient safety. Curr Opin Anaesthesiol 2015;28:685-90.

35 Brown M, Pinchin J, Valand R et al. NightShift simulation to train newly qualified doctors in non-technical skills: a feasibility study. Future Healthcare Journal 2016:3:94-8.

Address for correspondence: Dr Sophie Middleton, Thoracic medicine, University Hospital Aintree, Longmoor Lane, Liverpool L9 7AL, UK.

Email: sophie.middleton3@nhs.net 